\section{$\underset{\substack{\text { hommes } \\ \text { \& migrations }}}{ }$}

\section{Hommes \& migrations}

Revue française de référence sur les dynamiques

migratoires

\section{$1322 \mid 2018$}

Exposer les migrations

\title{
La « fabrique » des expositions sur les migrations
}

Un nouvel objet d'étude

\section{Helene Bertheleu, Piero Galloro et Mikaël Petitjean}

\section{(2) OpenEdition \\ 1 Journals}

\section{Édition électronique}

URL : https://journals.openedition.org/hommesmigrations/6589

DOI : 10.4000/hommesmigrations.6589

ISSN : 2262-3353

Éditeur

Musée national de l'histoire de l'immigration

Édition imprimée

Date de publication : 1 juillet 2018

Pagination : 9-16

ISBN : 978-2-919040-42-1

ISSN : $1142-852 X$

Référence électronique

Helene Bertheleu, Piero Galloro et Mikaël Petitjean, «La « fabrique » des expositions sur les migrations », Hommes \& migrations [En ligne], 1322 | 2018, mis en ligne le 01 juillet 2020, consulté le 21 janvier 2022. URL : http://journals.openedition.org/hommesmigrations/6589 ; DOI : https://doi.org/ 10.4000/hommesmigrations.6589 


\title{
$L A \ll F A B R I Q U E »$ DES EXPOSITIONS SUR LES MIGRATIONS UN NOUVEL OBJET D'ÉTUDE
}

Par HÉLÈNE BERTHELEU, sociologue, maître de conférences, université de Tours, CNRS-Citeres, PIERO GALLORO, maitre de conférences, HDR de sociologie, université de Lorraine et MIKAËL PETITJEAN, chargé de mission au Musée national de l'histoire de l'immigration.

\author{
Porté par des initiatives pionnières dès les années 1980, \\ le traitement muséographique des questions migratoires révèle \\ une dimension collaborative croissante dans la conception \\ des expositions. Ces projets d'expositions ont ainsi élaboré \\ leur propre méthodologie permettant de réunir des chercheurs, \\ des institutions patrimoniales et des associations. En recueillant \\ les témoignages et les traces des parcours migratoires, \\ il s'agit à la fois de faire connaître et reconnaître des histoires \\ de vie à l'échelle locale et de verser ces mémoires \\ au patrimoine commun. Une double tâche sur laquelle repose \\ une véritable démocratie patrimoniale.
}

Toute exposition est à considérer comme un dispositif communicationnel indissociable de son contexte de production. Support autant qu'intermédiaire entre ce qui est donné à voir et ce qui est vu, elle est aussi un révélateur de l'espace et du temps de sa création. Depuis les années 1980, l'exposition autour du thème des migrations, constitue une véritable mise en scène objectivée des subjectivités et des affects, faisant émerger une lecture tant de l'objet lui-même que de la société qui l'appréhende. Les dispositifs de création des expositions sur les migrations deviennent alors un nouvel objet d'étude que nous proposons d'appréhender à travers trois entrées : la reconnaissance, la performativité et la dimension artistique. Il convient de les comprendre à partir de situations diverses et en confrontant les points de vue des différents acteurs engagés dans ces expositions : les professionnels du patrimoine, les associations, les chercheurs, les artistes et scénographes et, souvent aussi, les acteurs du champ social.

\section{Exposition : une contribution historique à la reconnaissance?}

La notion de reconnaissance s'articule aujourd'hui à celle de justice sociale dans un contexte non plus tant de lutte contre l'exploitation - comme l'exhortaient les idéologies dominantes jusqu'à la fin du $\mathrm{XX}^{\mathrm{e}}$ siècle - mais de réaction à la domination culturelle ${ }^{1}$. En France, depuis les années 1980, la lutte 
pour la reconnaissance des identités occupe l'espace public au même titre que celle de l'injustice socioéconomique. On constate d'ailleurs que la question de l'immigration surgit au moment des luttes contre la désindustrialisation des années 1980. Aux mobilisations politiques contre l'injustice de l'exploitation et de la redistribution des richesses matérielles, s'ajoute celle en faveur d'une reconnaissance culturelle et identitaire. Or il ne s'agit pas

L'exposition Les enfants de l'immigration, présentée en 1984 au Centre Pompidou,

témoigne de façon emblématique de

cette politique et constitue

le premier programme

culturel de cette envergure dédié à l'immigration.

raine ${ }^{3}$ en sociologie 4 et en géographie ${ }^{5}$ posent les jalons d'un champ historiographique qui se développe dans les années 1980, d'abord à la périphérie de l'histoire ouvrière ou de l'histoire de l'opinion publique $^{6}$, puis grâce à des synthèses ${ }^{7}$. L'émergence de ce courant historiographique est concomitante de l'écho grandissant de revendications pour faire reconnaitre les droits des « immigrés ${ }^{8}$ » et leur place au sein de la société française. La Marche pour l'égalité et contre le racisme à l'automne 1983 en est la forme la plus médiatique ${ }^{9}$. Militants et chercheurs trouvent donc un terrain de travail commun lorsqu'il s'agit de faire reconnaître la place de l'immigration dans l'histoire de France. Dans un premier temps, les acteurs patrimoniaux demeurent largement à l'écart de ce mouvement.

Les politiques d'accueil et d'intégration des immigrés évoluent fortement dans les années 1980. La valorisation des cultures d'origine en vue de favoriser les « retours au pays » est partiellement remise en question. À partir de 1982, une meilleure connaissance des cultures est encouragée pour lutter contre l'intolérance et le racisme ${ }^{10}$. Elle se traduit par des actions linguistiques et interculturelles. Le ministère de la Culture élargit son périmètre d'action pour "préserver le patrimoine culturel national, régional ou des divers groupes sociaux pour le profit commun de la collectivité tout entière ${ }^{11} »$. L'exposition Les enfants de l'immigration, présentée en 1984 au Centre Pompidou, témoigne de façon emblématique de cette politique et constitue le premier programme culturel de cette envergure dédié à l'immigration ${ }^{12}$. Il est d'ailleurs revendiqué comme une étape fondatrice par certains acteurs, comme l'association Aidda ${ }^{13}$. Une telle initiative demeure isolée et les alternances politiques conjuguées aux réticences du ministère de la Culture pondèrent cette dynamique dès 1986. Cependant, cette impulsion trouve néanmoins d'autres relais au sein du secteur culturel avec l'essor de la nouvelle muséologie. Dans la continuité des travaux de GeorgesHenri Rivière et d'Hugues de Varine, plusieurs acteurs, comme le Musée d'histoire de Marseille,

\footnotetext{
2. Se référer notamment au rapport "La recherche sur les migrations et l'immigration. Un état des lieux ", Musée national de l'histoire de l'immigration, mars 2017. 3. Michelle Perrot, "Les rapports entre ouvriers français et étrangers (1871-1893) ", in Bulletin de la Société d'histoire moderne, $\mathrm{n}^{\circ} 12,1960$, pp. 4-9 ; Jean-Charles Bonnet, Les pouvoirs publics français et l'immigration dans l'entre-deux-guerres, Lyon, Centre d'histoire économique et sociale de la région lyonnaise, 1976. 4. Alain Girard, Jean Stoetzel, "Français et immigrés. L’attitude française. L'adaptation des Italiens et des Polonais ", in Travaux et documents, n 19, Ined, 1953. 5. Anne-Marie Fixot, "L'immigration de travailleurs saisonniers dans le Vaucluse. Étude de géographie rurale ", Mémoire de maîtrise, Caen, Université de Caen, 1970. 6. Gérard Noiriel, Longwy, Immigrés et prolétaires (1880-1980), Paris, Presses universitaires de France, 1984 ; Ralph Schor, L'opinion française et les étrangers en France, 1919-1939, Paris, Publications de la Sorbonne, 1985. 7. Pierre Milza, Marianne Amar, L'immigration en France au XXe siècle, Paris, Armand Colin, 1990 ; Gérard Noiriel, Le creuset français. Histoire de l'immigration (XIXe-XXe siècle), Paris, Seuil, 1988. 8. Le terme "immigré " appartient au langage commun des années 1960-1970, sous la forme d'abord de " travailleurs immigrés " puis d' "immigrés " à partir des années 1980. Les chercheurs s'expriment alors plutôt en utilisant la distinction juridique étrangers/nationaux, considérant les usages sociaux de « immigrés " plus péjoratifs que descriptifs. Le terme " immigré » est finalement adopté par l'Institut national d'études démographiques (Ined) en 1992, devenant une catégorie officielle de la statistique française. En 1992, l'Ined lance en effet l'enquête "Mobilité géographique et insertion sociale " dans laquelle est retenue la catégorie " immigrée " plutôt qu'étranger. Voir Michèle Tribalat, Faire France : une grande enquête sur les immigrés et leurs enfants, Paris, La Découverte, 1995. 9. Voir à ce propos le numéro d'Hommes \& Migrations, $n^{\circ} 1313$, consacré à " 1983, le tournant médiatique ". 10. Voir le rapport remis au gouvernement en 1982 par la députée Françoise Gaspard L'information et l'expression culturelle des communautés immigrées en France, qui pose les jalons de cette politique davantage inclusive. 11. Se rapporter notamment à Angéline Escaffré-Dublet, Immigration et politiques culturelles, Paris, La documentation française, 2014. 12. Adèle Momméja, "Les enfants de l'immigration au Centre Pompidou ", in Hommes \& Migrations, $n^{\circ}$ 1313, 2016, pp. 97-102. 13. Créée en 1985, l'Association interculturelle de production de diffusion et de documentation audiovisuelle (Aidda) promeut la photographie sociale et documentaire. URL : www.aidda.com (consulté le 07/03/2018).
} 
l'Écomusée de Fresnes ou encore le Musée dauphinois militent pour que le musée devienne un acteur social, ouvert à son territoire et à l'ensemble de sa population. Pour ces professionnels du patrimoine, les habitants dans toute leur diversité, immigrés ou non, deviennent la préoccupation principale du musée, à la fois acteurs de la vie du musée et public potentiel auquel il faut s'adresser.

La création de l'association Génériques en 1987, suivie, deux ans plus tard, de l'exposition 1789-1989. France des étrangers, France des libertés pose un jalon important. Pour la première fois, une exposition est pleinement dédiée à l'histoire de la présence immigrée en France. Historiens, militants associatifs et structures patrimoniales collaborent à la réalisation de cette exposition qui est présentée au Musée d'histoire de Marseille dans le cadre des commémorations du bicentenaire de la Révolution française ${ }^{14}$.

En 1990, une association œuvrant pour la création d'un musée français de l'immigration est créée par plusieurs personnalités universitaires et associatives, sans suite immédiate. Le faible nombre d'expositions proposées sur cette thématique dans les années 1990 contraste avec l'apparition d'acteurs associatifs décidés à réaliser un travail historique et mémoriel de la migration qu'ils représentent, comme la Fédération des associations et centres d'Espagnols émigrés en France, créée en 1991. 
La réalisation de l'exposition Toute la France! Histoire de l'immigration en France au XX $X^{e}$ siècle par la Bibliothèque de documentation internationale contemporaine (BDIC) et la Ligue de l'enseignement en 1998-1999 marque une nouvelle étape. Présentée au cœur de Paris au sein du Musée d'histoire contemporaine, dans l'Hôtel des Invalides, cette exposition succède involontairement à la célébration de l'équipe de France «blackblanc-beur » vainqueur de la Coupe du monde de football à l'été 1998.

\section{Le temps de la structuration: les années 2000}

Les élections présidentielles de 2002 remettent la question de l'histoire de l'immigration au goût du jour, comme en témoigne la création de la Cité nationale de l'histoire de l'immigration (CNHI), renommée en 2013 « Musée national de l'histoire de l'immigration ». Ouverte en octobre 2007 après quatre années de préfiguration, la CNHI prend la forme d'un musée national, actant une reconnaissance institutionnelle du patrimoine de l'immigration au sein du patrimoine national. Les difficultés rencontrées dans les premières années témoignent de l'ampleur du travail à conduire pour que cette reconnaissance dépasse le cadre institutionnel.

Cette création s'inscrit dans une dynamique des opérateurs et des administrations de l'État portée par les recommandations du comité interministériel à l'intégration réuni en 2003. En 2006-2008, le Fasild ${ }^{15}$, puis l'Agence nationale pour la cohésion sociale et l'égalité des chances (Acsé) impulsent et financent une série de rapports sur l'histoire de l'immigration dans les régions, afin de produire des connaissances territorialisées sur le sujet. Simultanément, le ministère de la Culture, par le biais de sa Mission à l'ethnologie, lance un programme de recherche "Mémoire de l'immigration, vers un processus de patrimonialisation ». L'Institut national de la recherche pédagogique publie pour sa part un rapport sur l'enseignement de l'histoire de l'immigration ${ }^{16}$. Des associations sont créées en régions pour fédérer les acteurs dans les territoires, à l'image du réseau aquitain pour l'histoire et les mémoires de l'immigration en 2004. Les projets d'exposition sur l'histoire de l'immigration se multiplient, le plus souvent dans un format " exposition panneaux ». Entre 2003 et 2009, un premier repérage non exhaustif permet d'identifier 105 présentations ${ }^{17}$. Durant cette période, les musées et les centres d'archives demeurent encore largement à l'écart de cette dynamique.

En réorientant les actions de l'Acsé vers la politique de la ville en 2009, l'État amorce un mouvement de désengagement du champ de l'histoire et la mémoire de l'immigration. Une ligne budgétaire intitulée "histoire/mémoire » demeure dans la politique de la ville (connue sous le sigle « bop147»), mais est clairement fléchée sur l'histoire des quartiers prioritaires. Les projets histoire/mémoire de l'immigration plus transversaux restent financés par la politique d'intégration (bop104) jusqu'en 2013. L'évolution de la politique d'intégration en direction des "primo-arrivants », c'est-à-dire des personnes arrivées en France depuis moins de cinq années, met fin à ce financement.

Ce contexte budgétaire contraint n'empêche pas une structuration progressive des réseaux associatifs au niveau régional. Certaines activités poursuivent leur développement, comme les expositions itinérantes destinées à des équipements socioculturels ${ }^{18}$. Dans les territoires, les collectivités se mobilisent progressivement sur cette thématique, particulièrement à travers leurs services patrimoniaux, archives et musées. Les expositions sont le témoignage de cette mobilisation : non exhaustive, une première recherche conduite au sein du MNHI 
a permis l'identification d'environ 200 «expositions » entre 2009 et $2017^{19}$.

Plus exceptionnellement, certains projets patrimoniaux se cristallisent autour de cette thématique, à l'image du Centre du patrimoine arménien à Valence. D'autres intègrent la thématique migratoire au cœur de leur projet scientifique et culturel, à l'image du travail engagé par le Musée de Barcelonnette depuis plusieurs années.

\section{Exposition, participation et performativité}

La multiplication de ces expositions signale une montée en visibilité patrimoniale. Elle interroge également la place que prennent les populations " concernées » dans ces projets. Comment les institutions patrimoniales travaillent-elles avec la diversité des acteurs de leur territoire? On peut aussi s'interroger sur les effets politiques de ces initiatives : chaque exposition suppose que les acteurs s'entendent sur son message principal, sur les publics auxquels on veut s'adresser, sur les stratégies scénographiques qui serviront le mieux les objectifs du projet : les populations concernées parviennentelles, au cours de ces différents moments, à prendre la parole et à sortir de la discrétion à laquelle les « immigrés » ont longtemps été assignés ?

La muséologie contemporaine est influencée par l'expérience des écomusées qui, à partir des années 1970, ont interrogé la mission du musée, remettant en cause le projet patrimonial d'institutionnalisation verticale et descendante du patrimoine. Les écomusées invitent à s'ouvrir aux territoires, à puiser des forces dans les caractéristiques sociales et culturelles de la population, à refléter cette diversité et ainsi à élargir les publics. Dans cette démarche, on l'a dit, le Musée dauphinois fait figure de pionnier en se tournant délibérément, dès la fin des années 1980, vers les migrations qui ont marqué l'histoire démographique et sociologique de l'Isère. Cette considération pour la population

locale se traduit aussi par une méthode de travail qui associe étroitement les associations dites « communautaires » à la confection de l'exposition.

Cette démarche rompt avec les représentations habituelles et médiatiques des migrations qui les envisagent au mieux comme participant au développement économique, au pire comme des problèmes sociaux qui pèsent sur la vie sociale du pays. Considérer ce long passé migratoire comme mémorable et ces histoires migratoires comme susceptibles de constituer un patrimoine, constitue un défi pour les professionnels du patrimoine et les associations, perçus comme des militants lorsqu'ils décident de faire une exposition sur les migrations. Ils interrogent en effet le compromis républicain qui, jusque-là, avait choisi de couvrir ces questionnements d'un voile silencieux. Les professionnels des institutions patrimoniales qui

La muséologie contemporaine est influencée par l'expérience des écomusées qui, à partir des années 1970, ont interrogé la mission du musée, remettant en cause le projet patrimonial d'institutionnalisation verticale et descendante du patrimoine. s'engagent alors dans ce type de projet ne sont pas nombreux et peuvent rencontrer des réticences diverses, allant du désintérêt de leurs collaborateurs et techniciens, en passant par la prudence ou l'inquiétude des tutelles jusqu'à la réaction xénophobe de certains groupuscules au moment de l'ouverture au public.

Une autre difficulté réside dans l'ambivalence des concernés eux-mêmes, les migrants ou leurs descendants. Beaucoup sont réticents à «exposer » leur vécu à autrui, à rendre visible une expérience intime, marquée par des difficultés voire des humiliations. Certains, dans un autre registre, s'interrogent sur les effets politiques d'une telle prise de parole, dans un contexte français encore largement assimilationniste, malgré la récurrence des débats sur la diversité culturelle ou le multiculturalisme. D'autres, au contraire, anticipent les bénéfices sociaux de cette reconnaissance publique mais n'en redoutent pas moins le manque de maîtrise du récit collectif qui sortira de ce travail d'«exposition ${ }^{20}$ ". 
Les témoignages collectés sont diffusés dans l'exposition Lorrains sans frontières. C'est notre histoire! Photo Michel Bourguet. (c) Palais des Ducs de lorraine-Musée lorrain, Nancy.

Enfin, au-delà de tous ces éléments envisagés, l'exercice reste périlleux : n'est-il pas vain ou hasardeux de vouloir publiciser des mémoires si diverses et complexes? Ne risquent-elles pas d'être tronquées, réduites et «neutralisées » par le processus d'exposition, voire de patrimonialisation? En voulant raconter des histoires et des souvenirs, l'exposition risque de trahir l'authenticité de ces derniers.

\section{Les défis des expériences collaboratives}

Aujourd'hui, répondant en partie à ces questionnements, les projets d'exposition mettent souvent en œuvre des formes variées de collaboration : les institutions patrimoniales s'associent par exemple à des chercheurs de terrain ou se tournent vers des artistes chargés de recueillir la parole des concernés et d'incarner par leurs œuvres la complexité des trajectoires migratoires. Le répertoire des actions menées dénote la multiplication des formes, des coopérations, et l'importance du développement de réseaux locaux ou nationaux. Ce fut ainsi l'objectif du Groupement d'intérêt scientifique «Institutions patrimoniales et pratiques interculturelles » porté notamment par Hélène Hatzfeld, alors chargée de mission au ministère de la Culture, qui a travaillé durant plusieurs années à favoriser et valoriser partout en France les expériences d'hybridation des regards ${ }^{21}$, incitant les professionnels à sortir de leurs compétences et leurs terrains habituels pour s'aventurer vers des collaborations avec des mondes sociaux méconnus. 
Au-delà de ces expériences, il faudra s'interroger sur la portée de ces initiatives, sur leurs effets à plus long terme. Elles participent sans doute à une meilleure connaissance des populations issues de ces migrations, à leur ancrage au sein des territoires ${ }^{22}$. Cela ne signifie pas que les mémoires collectives ainsi reconnues au sein des institutions patrimoniales se cristallisent finalement en un patrimoine commun. Le processus de patrimonialisation est long et complexe : beaucoup d'expositions restent éphémères et s'appuient sur des prêts, ne débouchant donc ni sur des acquisitions ni sur des collections.

Un des défis de ces expériences collaboratives renvoie à la participation des populations directement concernées par les migrations. Comment cette participation est organisée, mise en œuvre concrètement? Quelles sont finalement les personnes autour de la table, non seulement pour contribuer à la collecte, mais aussi, plus fondamentalement, pour discuter des visées de l'exposition? Dans un certain nombre de cas, l'ouverture du projet à des personnes extérieures se limite à la " participation » de témoins, dont le pouvoir n'excède pas le contenu et la mise en scène de leurs propres témoignages. Parfois, la collaboration va plus loin mais interroge alors le pouvoir et la temporalité de l'institution patrimoniale. Est-elle prête à partager, avec les concernés, la conception de l'exposition, la discussion du synopsis, la forme dans et hors les murs, etc., jusqu'aux arbitrages budgétaires? Ici comme ailleurs, la notion de participation est polysémique $^{23}$ et fait l'objet d'appropriations variées. En France, la méthode participative confine parfois à l'injonction, au risque de se réduire à une sémantique institutionnelle dont on attend surtout les effets de légitimation aux yeux des pouvoirs publics soutenant le projet.

Il faudra du temps pour mesurer le succès de ces expositions, non pas seulement en termes de nombre de visiteurs, mais plutôt par leur capacité à affirmer et mettre en œuvre une démocratie patrimoniale. Les effets, la performativité d'une exposition ne se mesure qu'à long terme : l'exposition Toute la France portée en 1998 par la Bibliothèque de documentation internationale contemporaine (BDIC), on peut le dire aujourd'hui vingt ans plus tard, a entraîné de nouveaux partenariats, a contribué à transformer les représentations, a initié une légitimité de l’objet « immigration » qui a permis une progressive visibilité.

Pour de nombreux anciens Le processus de migrants, partenaires actifs de tous ces projets culturels et patrimoniaux, le processus de reconnaissance ne fait pas de doute et accompagne le sentiment et l'affirmation d'une certaine autochtonie. Néanmoins, patrimonialisation est long et complexe : beaucoup d'expositions restent éphémères et s'appuient sur des prêts, ne débouchant donc ni sur des acquisitions ni sur des collections.

histoire passée longtemps restée invisible, celle de familles immigrées venant désormais "pluraliser » la représentation de la population locale, d'autres vont surtout y voir du territoire, des habitants et de l'intégration. L'exposition est la même mais ils n'y lisent pas la même histoire et, finalement, n'y valident pas le même patrimoine.

\section{Exposer, rendre visible, montrer}

Quoi qu'il en soit, les expositions travaillent à rendre visibles des immigrations en France dans l'espace public à travers la dimension historique et patrimoniale. Si les travaux scientifiques ont permis, pour leur part, de décrypter les mécanismes racisants et d'exclusion sociale, leur diffusion auprès du grand public et leur capacité à influer sur les mentalités restent limitées. Parallèlement à cette prise de conscience des chercheurs du faible écho de leurs travaux dans l'espace public, les artistes ont aujourd'hui affirmé leur rôle et leur capacité à articuler enjeux politiques et esthétisation des phénomènes de société. Depuis les années 1970, le débat porte moins sur les figures de l'art et de la science que sur leurs conditions de 
production et de réception. L'œuvre artistique in situ (land art, théâtre invisible, street art, etc.) a réussi à déplacer la création artistique de lieux spécialisés vers l'espace public. Les artistes se nourrissent des connaissances scientifiques et les traduisent en émotions. Artistes et scientifiques ont réalisé qu'ils ne vivaient pas dans des mondes étanches et qu'ils étaient les uns comme les autres en prise avec les réalités sociales. Chacun, dans son œuvre respective, questionne son environnement, élabore une problématique, construit des outils pour y répondre et valorise le résultat de sa réflexion. Au-delà de leurs langages distincts, artistes et chercheurs produisent des questionnements et des émotions, pointent des dysfonctionnements sociaux, revisitent les savoirs établis et apportent un certain regard sur le monde. Une véritable proximité lie chercheurs et artistes par les modalités de travail, de réflexion et/ou d'engagement social.

Pour conclure sur les expositions elles-mêmes, il convient de souligner le caractère polysémique du média lui-même, mais aussi la polyphonie des propos recueillis auprès des chercheurs, artistes et/ou militants qui se sont efforcés de re-travailler le regard sur les migrations en termes de démonstration de la mise en scène médiatique qui, de "crises des migrants » en « jungle», donne une vision alarmiste des migrations et catastrophiste de l'intégration culturelle des "étranges-étrangers ${ }^{24}$ ». Montrer, c'est avant tout désigner, terme qui, dans son origine latine "designare», signifie "marquer d'un signe». Ce marquage permet d'indiquer (de "montrer du doigt », c'est-à-dire de "mettre à l'index »), d'attirer l'attention, de signaler (là encore, la racine latine « signum » renvoie au signe) quelque chose ou quelqu'un. Autrement dit, la monstration permet de signifier, de marquer pour mieux distinguer et séparer par les voies du spectaculaire: le spectacle désigne étymologiquement «ce qui parle aux yeux de l'imagination " (Le Robert, 2017). Ainsi, dans la préface aux Damnés de la terre de Frantz Fanon, Jean-Paul Sartre, pour mieux dénoncer la construction de l'altérité de l'" Autre », écrivait que "l'Européen, n'a pu devenir Homme qu'en créant des monstres ${ }^{25} »$. Il est nécessaire d'interroger les mécanismes mis en œuvre qui permettent de «mieux comprendre certaines pratiques sociales, de retracer l'évolution du concept d'anormalité et de théoriser le regard que nous portons sur la différence ${ }^{26}{ }$.

Finalement, l'exposition est intéressante non parce qu'elle permettrait de souligner les différences mais parce qu'elle donne à voir du commun dans un rapport de banalité. Dans le sens étymologique, le banal c'est justement ce qui est "commun». D'abord dans son premier sens de trivial, d'ordinaire. Un second sens, plus riche, renvoie selon les dictionnaires à l'idée de participation collective - tout le monde peut en profiter - comme dans les expressions "le commun des mortels", «le droit commun", "le dénominateur commun", etc. Exposer les (im)migrations c'est donc aussi, en quelque sorte, montrer et susciter une participation collective qui dépasse le clivage nativiste (séparant les immigrés et leurs héritiers des populations imaginées de souche), en privilégiant l'idée d'ensemble, de conjoint, d'inclusion, c'est-à-dire du " commun » comme dans faire "cause commune", "avoir quelque chose en commun ", «l'intérêt commun ». Cette définition du commun se rattache à l'origine latine "communis » qui désigne les personnes qui supportent ensemble (cum) les charges (munus). Or cette conformité n'a rien de naturel puisqu'elle est le produit de l'action humaine. En cela, la banalité apparaît comme le contenu d'une certaine forme de regard porté sur le réel, comme un produit d'opérations qu'il semble naturel à l'homme d'accomplir. Par-là, la démarche d'exposition revient à changer les regards pour mieux révéler le banal et le commun et, ainsi, réinsérer dans le tissu social ce qui en était détaché et qui se dérobait aux formes d’appréciation ordinaires. 